\title{
Bio-inspired Aging Model-Particle Swarm Optimization and Geometric Algebra for Structure from Motion
}

\author{
Nancy Arana-Daniel, Carlos Villaseñor, \\ Carlos López-Franco, and Alma Y. Alanís \\ Universidad de Guadalajara \\ Centro Universitario de Ciencias Exactas e Ingenierías \\ Blvd. Marcelino García Barragán \# 1421, Guadalajara, Jalisco, México
}

\begin{abstract}
On computer vision field Structure from Motion (SfM) algorithms offer good advantages for numerous applications (augmented reality, autonomous navigation, motion capture, remote sensing, object recognition, image-base $3 \mathrm{D}$ modeling, among others), nevertheless, these algorithms show some weakness; in the present paper we propose the use of Bio-inspired Aging Model-PSO (BAM-PSO) to improve the accuracy of SfM algorithms. The BAM-PSO algorithm is used over a Geometric Algebra (GA) framework in order to compute the rigid movement on images and this allows us to obtain a numerically stable algorithm.
\end{abstract}

Keywords: SfM, Conformal Geometric Algebra, PSO.

\section{Introduction}

Computer vision has been a very active research field in the last decades, but the problems to be solved have proven to be difficult. Nevertheless, new paradigms improve the robot capabilities in the field. The present paper proposes the use of evolutionary computation over a geometric mathematical framework to improve some aspects of Structure from Motion (SfM) Algorithms.

Structure from Motion represents a family of computer vision algorithms whose paradigm is based on extracting structures when movement is detected or extracting movement when structures are detected using 2D correspondences from multiple images, 73. There are several approaches for SfM algorithms, for example, the use of monocular camera systems for 3D reconstructions [10] or segmentation from multi-body motion [10].

Most of two view SfM algorithms are based on the epipolar restriction (Eq. 1) between at least, two images of the same scenario: image- $i$ and image- $j$

$$
x^{\prime} E x=0
$$

where $E=\hat{T} R$ is called the essential matrix (i.e. the matrix which relates corresponding points in stereo images assuming that the cameras satisfy the pinhole camera model), therefore $E$ represents the rigid motion between image- $i$

E. Bayro-Corrochano and E. Hancock (Eds.): CIARP 2014, LNCS 8827, pp. 762769 2014.

(C) Springer International Publishing Switzerland 2014 
and image- $j, \hat{T}$ is the skew-symmetric matrix that contains the translation and $R$ is the rotation matrix , $x$ stands for a point on image- $i$, while $x^{\prime}$ represents the corresponding point for $x$ on image- $j$. The computation of this matrix can be hard to achieve, and this is the main difference between SfM algorithms.

Some SfM algorithms compute the essential matrix directly using for example an 8-point algorithm, the result is limited by the calibration of the camera, [7]. Most of SfM compute the fundamental matrix through 8-point, RANSAC or others algorithms [7, [13, and they get a weak perspective bifocal tensor, after that, we can compute the essential matrix with the intrinsic parameters, or use a self-calibration technique. Other SfM algorithms use trifocal ([2]) or n-focal tensors $([12,3])$ to get a stronger perspective which is very useful, although, they are computationally expensive and they also depend on the calibration process.

In this work, we propose a novel algorithm to compute the movement which defines the essential matrix by using a variation of the evolutionary computation algorithm called Particle Swarm Optimization (PSO) [5] combined with the mathematical framework known as Geometric Algebra (GA) in order to find the motor (rotation and translation versor) that represents the motion between views. Evolutionary computation algorithms like PSO offer a fast convergence in solving oprtimization problems, and using them on SfM, can provide small reprojection errors respect to others reconstruction approaches.

The main task on PSO algorithms is to find a suitable objective function; using a mathematical framework such Geometric Algebra, we can address this problem with the advantage of using explicitly different geometric entities as 2D image correspondences (besides points, we can define planes, spheres, circles, etc. and their operators using the same algebra). By using geometric entities which are more complex than points we can access to richer information of the structure of the environments and therefore we can made more accurate $3 \mathrm{D}$ reconstructions as it is shown in Sec. 6. The use of GA framework also allows us to obtain a numerically stable algorithm: the computation of the rigid movement is done with a versor representation called motor $(M)$, this includes the rotation and translation movement in its definition, and it provides an uniquely representation of the (scaling) rotation transformation in 3D, which does not happen when Euler angles or matrix algebra are used, because the three Euler angles from which a rotation matrix can be constructed do not suffice by themselves [11.

This paper is organized as follows: In Sec. 2 is presented the evolutionary computation algorithm used in order to obtain the motion transformation between two views. In Sec. 3 it is given a brief introduction to Conformal Geometric Algebra (CGA). Sec. 4 shows a SfM algorithm. Sec. 5 presents our algorithm, proposed to solve SfM problems. In Sec. [6] we show the results obtained in reconstruction 3D environments using our approach and some comparatives with other SfM algorithms, while Sec. 7 is devoted to conclusions and future work. 


\section{Bio-inspired Aging Model-PSO}

Particle Swarm Optimization [5] is an evolutionary algorithm based on observations of bird flocks and insect swarm behavior that provides high convergence speed and shows excellent results solving many of modern engineering problems 8. The PSO algorithm consists of an iterative adaptation of set of multidimensional vectors called particles that communicate information among them as a swarm which provides a set of candidate solutions for an objective function. Recently, there has been an increasing interest on developing improvements to the PSO algorithm. These improvements intent to alleviate the premature convergence problem of the original PSO algorithm. In this work, it is used one of these new proposed algorithms called BAM-PSO [1] due to it has proved to solve the above mentioned problem and improve the accuracy of the results obtained with other PSO variant algorithms.

The BAM-PSO algorithm is an evolutionary computation algorithm based on the Aging Leader and Challengers (ALC-PSO) algorithm [9]. The basic idea of both algorithms is to include the aging process to each particle of the swarm. Meanwhile, ALC-PSO uses a linear aging model, BAM-PSO proposes to use a bio-inspired aging model.

Each particle in the swarm has a velocity $v_{i j}$ Eq. 2 and a position $x_{i j}$ Eq. 3 and these quantities are adjusted in each iteration of the algorithm as follows:

$$
\begin{gathered}
v_{i j}(t+1)=w * v_{i j}(t)+c_{1} R_{1}\left(p_{i j}(t)-x_{i j}(t)\right)+c_{2} R_{2}\left(\text { leader }+x_{i j}(t)\right) \\
x_{i j}(t+1)=x_{i j}(t)+v_{i j}
\end{gathered}
$$

where $i$ is the $i$ th particle of the swarm, $j$ is the $j$ th element of dimension problem, $t$ is the iteration counter, $w$ represents the inertial weight factor (constant), $R_{1}$ and $R_{2}$ are random, normalized and uniformly distributed values, $c_{1}$ and $c_{2}$ represent the social and cognitive parameter, $x_{i j}$ is the particle $i j$ position for the iteration $t ; x_{i j}(t+1)$ is the particle $i j$ position for $t+1$ iteration, $v_{i j}(t)$ is the particle $i j$ velocity for t iteration. $p_{i j}(t)$ represents the local best position for particle $i j$ in iteration $t$ and leader is the particle holding the best solution found by the swarm at iteration $t$.

\section{Conformal Geometric Algebra}

Geometric Algebra also known as Clifford Algebra, in fact represents a family of algebras that depend on a chosen vector space and on a special kind of product (the geometric product). The GA is a mathematical framework where we can find embedded concepts from linear algebra, tensor algebra, quaternion algebra, complex numbers and others. We represent a geometric algebra with $G_{p, q, 1} 1$.

The Conformal Geometric Algebra (CGA) is a 5-D Geometric Algebra that uses a conformal transformation to span euclidean geometric algebra represented

\footnotetext{
${ }^{1}$ For a more extensive introduction to Geometric Algebra please see [4], 11.
} 
by $G_{3}$, to $G_{4,1}$. One of the advantages of use more dimensions is that problems are intuitively and easily formulated.

In CGA we use the Euclidean basis vectors $e_{1}, e_{2}, e_{3}$ that we can span with a conformal transformation over the Minkowski's plane with the basis $e_{-}, e_{+}$that holds the Eq. 4. We can define another basis called null with Eq. 5

$$
\begin{aligned}
& e_{+}^{2}=-1, \quad e_{-}^{2}=1, \quad e_{+} \cdot e_{-}=0 \\
& e_{0}=\frac{1}{2}\left(e_{-}-e_{+}\right), \quad e_{\infty}=e_{-}+e_{+} .
\end{aligned}
$$

Where $e_{0}$ represents the $3 \mathrm{D}$ origin and $e_{\infty}$ represents the point to the infinity, they are null vectors and hold the Eq. 6

$$
e_{0}^{2}=e_{\infty}^{2}=0, \quad e_{\infty} \cdot e_{0}=-1
$$

With this basis, geometric entities are represented as multivectors, in fact, there is a dual representation for every entity; the inner product null space representation (IPNS) and the outer product null space representation (OPNS).

The basic geometric entities and their representations are listed in the Table 1. where $x_{E}$ stand for an euclidean point, $x_{C}$ for a conformal point, $\mathrm{r}$ for the radius of the sphere, $n$ a unitary vector and $d$ a distance from the origin (Hesse representation of the plane) and finally $\mathbf{n}$ and $\mathbf{m}$ stand for the Plucker coordinates of the line.

Table 1. Geometric entities on CGA

\begin{tabular}{|l|c|c|}
\hline Entity & IPNS & OPNS \\
\hline Point & $x_{C}=x_{E}+\frac{1}{2} x_{E}^{2} e_{\infty}+e_{0}$ & $x_{C}^{*}=s_{1} \wedge s_{2} \wedge s_{3} \wedge s_{4}$ \\
Sphere & $s=x_{E}-\frac{1}{2}\left(x_{E}-r\right)^{2} e_{\infty}+e_{0}$ & $s^{*}=x_{1} \wedge x_{2} \wedge x_{3} \wedge x_{4}$ \\
Plane & $P=n+d e_{\infty}$ & $P^{*}=x_{1} \wedge x_{2} \wedge x_{3} \wedge e_{\infty}$ \\
Line & $L=\mathbf{n} I_{E}-e_{\infty} \mathbf{m} I_{E}$ & $L^{*}=x_{1} \wedge x_{2} \wedge e_{\infty}$ \\
Circle & $C=s_{1} \wedge s_{2}$ & $C^{*}=x_{1} \wedge x_{2} \wedge x_{3}$ \\
\hline
\end{tabular}

We can use the same basis to represent rigid transformations, in general any conformal transformation can be expressed as Eq. 7.

$$
\sigma X^{\prime}=H X \tilde{H}
$$

where $X, X^{\prime} \in G_{4,1}, H$ is a versor and $\sigma$ a scalar, $\sigma$ is apply to ensure that the null cone is invariant under $H$.

Rotations are represented by Rotors and they are defined in form of Eq. 8 .

$$
R=e^{-\frac{\theta}{2} \boldsymbol{n}}
$$

where $\boldsymbol{n}$ represents the dual entity of the rotation axis and $\theta$ represents the rotation angle.We also define the translator in the form of Eq. 9. 


$$
T=1+\frac{1}{2} \boldsymbol{t} e_{\infty}
$$

$\boldsymbol{t}$ is the euclidean translation. The null cone is invariant under $R$ and $T$ so $\sigma=1$ then the rigid transformations are represented by Eq. 10.

$$
X^{\prime}=R X \tilde{R}, \quad X^{\prime}=T X \tilde{T} .
$$

We can use the last equations to express a rigid body motion, we denote such operator and the conformal transformation with Eq. 11.

$$
M=T R, \quad X^{\prime}=M X \tilde{M} .
$$

This equation of rigid movement can be applied on every entity of the algebra.

\section{Structure from Motion}

In this section, a SfM algorithm is shown as reference framework for our proposed algorithm (3]).

One important step in SfM is to solve the correspondence problem, but this is out scope of this work, therefore, the correspondences will be defined by a supervised method. The SfM algorithm is shown in Algorithm 1.

Algorithm 1. Structure from motion

Require: Correspondences of images, Calibration matrix $(K)$.

Ensure: Rotation and Translation between images.

1: Use the correspondences to calculate the fundamental matrix $F$ \{using RANSAC\}

2: Calculate the essential matrix with $E=K^{T} F K$

3: Decompose $E=\hat{T} R$ on its singular values $E=U \Sigma V^{T}$ \{to extract the movement

4: Use the $W=\left(\begin{array}{ccc}0 & -1 & 0 \\ 1 & 0 & 0 \\ 0 & 0 & 1\end{array}\right)$ or $W^{T}$ defined on 3] to extract the movement with $\hat{T}=V W \Sigma V^{T}$ and $R=U W^{-1} V^{T}$.

5: Select supervisely the correct movement from the fourth possible answers There are possibles solutions due use of $W$ or $W^{T}$, see [3]

\section{Bio-inspired Aging Model-PSO with Geometric Algebra for SfM}

Object mapping approach allows a compact representation of the environment, based in the idea that we have a map with geometric entities (that could be obtained by the Hough Transform as shown in [6]); it is possible to use BAMPSO over a CGA to estimate the motion of the entities.

To establish a search space for the BAM-PSO, we need to define the parameters on the rigid transform with the versor $M$ expressed with the Eq. 12 where 
$T$ stands for the translation (see Eq. 9) and $R_{x}, R_{y}$ and $R_{z}$ for the rotation on $x, y$, and $z$ axes respectively(see Eq. 8).

$$
M=T R_{z} R_{y} R_{x}
$$

In order to operate the $M$ versor we define $\alpha_{x}, \alpha_{y}$ and $\alpha_{z}$ as the rotation angle of $R_{x}, R_{y}$ and $R_{z}$ respectively and $t_{x}, t_{y}$ and $t_{z}$ as the euclidean translation on T.Therefore, the parameters of the search space can be define like Eq. 13.

$$
\chi=\left\{\alpha_{x}, \alpha_{y}, \alpha_{z}, t_{x}, t_{y}, t_{z}\right\}
$$

This search space will be bounded for the first three parameters by $[-\pi, \pi]$. Using the RMSE as error the fitness function for BAM-PSO is defined in Eq. 14.

$$
f_{O}=\min _{\chi} \sum_{k=1}^{q} \sqrt{\frac{\sum_{l=1}^{s}\left(P_{l}\left(E_{k}^{\prime}\right)-P_{l}\left(M E_{k} \tilde{M}\right)\right)^{2}}{n}}
$$

Where the function $P(\cdot): G_{4,1} \rightarrow \mathbb{R}^{n}$ extract the parameters of the geometric entities, $q$ and $s$ represent the number of entities in the object and the number of parameters in the entity respectively. For a conformal point, sphere, plane or line $P(\cdot)=\left\{x_{E}\right\},\{p\},\{n, d\}$ or $\{\mathbf{n}, \mathbf{m}\}$ respectively, (see Table 1). It is important to note that the objective function not only works with basic geometric entities, we can also define multi-entities as rigid objects.

\section{Results}

Consider the couple of images in Fig. 1 as the input for a SfM algorithm. For comparative purposes lets define 5 interest objects extracted from the human figure on the images: 1) Head and truck, 2) right arm, 3) left arm, 4) right leg, 5) left leg. For SfM we defined supervised correspondences of each object.

For the BAM-PSO for SfM it is needed to extract objects from Fig: 1 in a supervised way to get geometric entities and their correspondences (we use stereo vision to create a $3 \mathrm{D}$ cloud point) then, we can form the map of geometric entities shown in Fig: 2]a) using the GA framework and definitions shown in Tab. 1.

The results of BAM-PSO for the calculate of the motion is shown on Fig: 2] b) that manifest the error minimization over 200 generations, it's easy to see the fast convergence to a small error. Finally, on Tab: 2 is presented a comparative between BAM-PSO with CGA for SfM and classic SfM; measuring time, PSO error (error reached for BAM-PSO algorithm) and an error that stands for the accuracy of the method described in the Eq. 15 where $x_{i}$ is a new $3 \mathrm{~d}$ point in the object on the initial frame, $x_{f}$ the same point on the final frame and $\mathrm{H}$ stand for the homogeneous transform.

$$
E=\left\|x_{f}-H x_{i}\right\|
$$




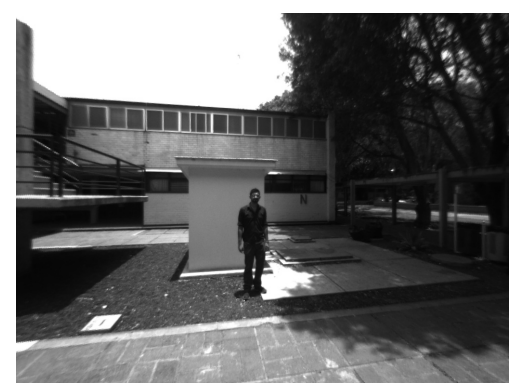

a)

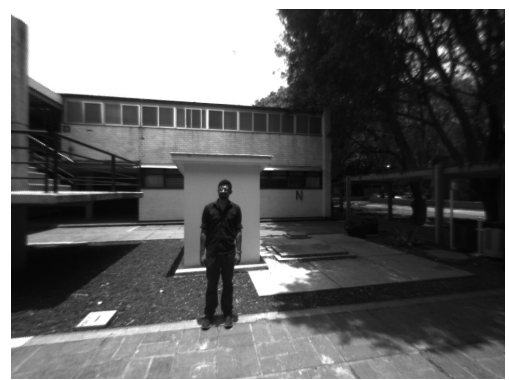

b)

Fig. 1. Input images

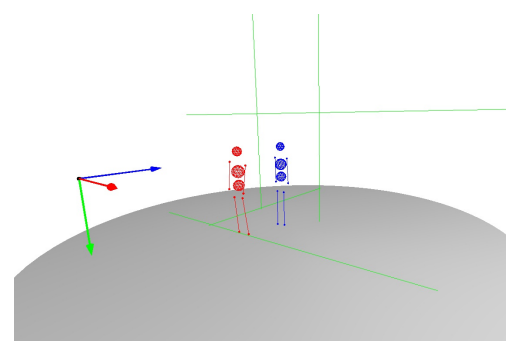

a)

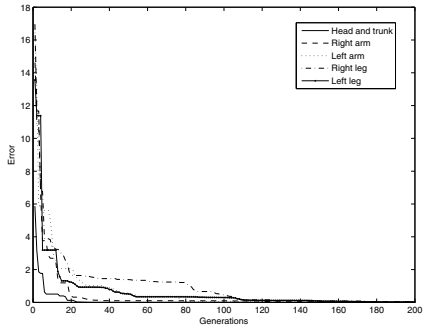

b)

Fig. 2. BAM-PSO. (a) Map of geometric entities, (b) Error function over generations.

Table 2. Comparative between BAM-PSO with CGA for SfM and classic SfM

\begin{tabular}{|l|c|c|c|c|c|}
\hline & \multicolumn{2}{|c|}{ BAM-PSO with CGA for SfM } & \multicolumn{2}{c|}{ Classic SfM } \\
\hline Object & Time (s) & PSO error & E (Eq: 15) & Time (s) & E (Eq: [15) \\
\hline Head and Trunk & 3.8165 & $2.2087 \mathrm{e}-04$ & 0.0002 & 2.1451 & 1.4072 \\
Right arm & 2.0896 & 0.0421 & 0.0094 & 2.0855 & 1.08575 \\
Left arm & 5.8751 & 0.0372 & 0.0103 & 2.0468 & 1.9581 \\
Right leg & 4.5828 & 0.0164 & 0.5854 & 1.9643 & 3.5366 \\
Left leg & 6.2066 & 0.0266 & 0.1984 & 1.9201 & 3.8789 \\
\hline
\end{tabular}

\section{Conclusions and Future Work}

As we already proved, the use of BAM-PSO over geometric entities represented on CGA improve the accuracy of SfM algorithms, the use of entities that are richer of structure information provides an error decrease and numerical stability for SfM. The future work based in this paper will include the parameterization of the circle (As the only entity missing on Sec. 5 and the process automation with hough transform as is shown on [6]. Also a parallelization of BAM-PSO algorithm will be implemented in order to reduce the processing time of SfM. 


\section{References}

1. Hernandez-Vargas, E.A., Lopez-Franco, C., Rangel, E., Arana-Daniel, N., Alanis, A.Y.: Particle swarm optimization with bio-inspired aging model. Submitted to IEEE Trans. Evolutionary Computation (2014)

2. Hartley, R., Vidal, R.: The multibody trifocal tensor: Motion segmentation from 3 perspective views. In: Proceedings of the 2004 IEEE Computer Society Conference on Computer Vision and Pattern Recognition, CVPR 2004, vol. 1, pp. I-769. IEEE (2004)

3. Hartley, R., Zisserman, A.: Multiple view geometry in computer vision. Robotica $23(2), 271-271(2005)$

4. Hildenbrand, D.: Foundations of Geometric Algebra Computing, vol. 8. Springer (2012)

5. Kennedy, J., Eberhart, R.C.: Particle swarm optimization. In: Proceedings of the IEEE International Conference on Neural Networks, pp. 1942-1948 (1995)

6. López-González, G., Arana-Daniel, N., Bayro-Corrochano, E.: Conformal hough transform for 2D and 3D cloud points. In: Ruiz-Shulcloper, J., Sanniti di Baja, G. (eds.) CIARP 2013, Part I. LNCS, vol. 8258, pp. 73-83. Springer, Heidelberg (2013)

7. Ma, Y., Soatto, S., Kosetska, J., Sastry, S.: An invitation to 3-D vision: from images to geometric models, vol. 26. Springer (2004)

8. Vrahatis, M.N., Parsopoulos, K.E.: Particle swarm optimization and intelligence, advances and applications. IGI Global (2010)

9. Chen, W.N., Zhang, J., Lin, Y., Chen, N., Zhan, Z.H., Chung, H.S.-H., Li, Y., Shi, Y.H.: Particle swarm optimization with an aging leader and challengers. IEEE Trans. Evolutionary Computation 17(2), 241-258 (2013)

10. Ozden, K.E., Schindler, K., Van Gool, L.: Simultaneous segmentation and 3D reconstruction of monocular image sequences. In: IEEE 11th International Conference on Computer Vision, ICCV 2007, pp. 1-8. IEEE (2007)

11. Christian, B., Perwass, U.: Geometric algebra with applications in engineering. Geometry and Computing, vol. 4. Springer, Heidelberg (2009)

12. Schindler, K., James, U., Wang, H.: Perspective n-view multibody structure-andmotion through model selection. In: Leonardis, A., Bischof, H., Pinz, A. (eds.) ECCV 2006, Part I. LNCS, vol. 3951, pp. 606-619. Springer, Heidelberg (2006)

13. Torr, P.H.S.: A structure and motion toolkit in matlab-interactive adventures in $\mathrm{S}$ and M. Microsoft Research (2002) 